Journal of Universal Mathematics

Vol.4 No.2 PP.259-270 (2021)

ISSN-2618-5660

DOI: $10.33773 /$ jum. 886932

\title{
BIPOLAR SOFT ORDERED TOPOLOGY AND A NEW DEFINITION FOR BIPOLAR SOFT TOPOLOGY
}

\author{
NAIME DEMIRTAŞ AND ORHAN DALKILIÇ \\ 0000-0003-4137-4810 and 0000-0003-3875-1398
}

\begin{abstract}
In our study, we gave a new definition for bipolar soft topology and we were able to examine the concept of bipolar soft ordered topology using the base concept we defined on this new bipolar soft topology. We also define the concept of bipolar soft set relation by defining an $\mathrm{R}$ relation on a bipolar soft set. Thus, we have defined the concept of bipolar soft interval and presented the bipolar soft ordered topology structure using these intervals in our study.

Then, we expressed some applications of bipolar soft order topology.
\end{abstract}

\section{INTRODUCTION}

Traditional methods fail to solve many complex problems especially in decision making due to uncertainty problems encountered in fields such as economy, engineering, environment. One of the theories put forward to eliminate uncertainty is the soft (briefly s-)set theory introduced by Molodtsov [2]. Then, Maji et al. [1] introduced some new concepts such as subset and complement to s-set theory. The studies on this set theory are increasing and there are many applications especially on s-sets in recent years $[8,11,12,13]$.

In 2010, Babitha and Sunil [14] defined the relation and the ordering in s-sets. Moreover, Park et. al [15] studied the equivalence relations, partitions and functions. In the following years, the notions of symmetric kernel, anti-reflexive kernel, symmetric clousure and reflexive clousure of a s-set relationship was given by Yang and Guo [16] and they proposed s-set relation mappings and inverse s-set relation mappings. The definition of supremum and infimum of the s-set, directed complete s-set were given by Tanay and Yaylalı [17].

The topology structure of s-set has been studied by many researchers and different definitions have been made: Shabir and Naz [18] introduced the soft topological spaces. They studied many concepts such as s-open set, s-neighbourhood of a point in s-topological spaces. As a different approach to s-topology; Cağman et al. [19] defined the concepts of s-closure, s-Hausdorff space, s-limit point, s-interior, s-open

Date: Received: 2021-02-25; Accepted: 2021-07-29.

2000 Mathematics Subject Classification. 03E72; 54E99.

Key words and phrases. bipolar soft ordered set, bipolar soft topology, bipolar soft ordered topology. 
set and the structure of s-topology was improved by Roy and Samanta [8]. In addition, many researchers such as Aygünoğlu and Aygün [20], Min [24], Zorlutuna et al.[21], Hussain and Ahmad [22], Varol and Aygün [23] studied s-topological spaces.

In last years, the concept of bipolar soft (briefly bs-)set defined by Shabir and Naz [25] and Karaaslan and Karatas[26]. After this set theory has been proposed, the structure of bs-topology has been defined by many researchers: Shabir M. and Bakhtawar A.[27] introduced the bs-topological spaces. Then, Öztürk Y.T.[28] introduced some properties of the bs-topological space.

In our study, we first defined a new bs-topological structure. Using this definition, we gave the concepts of bs-base and bs-intervals on this structure. Then, the bs-order topology is introduced and some applications of bs-order topology are expressed. At the same time, examples are added for easy understanding of the concepts and structures given in our study.

\section{Preliminaries}

In this section, we recall some basic notions in s-sets and bs-sets. Let $U$ be an initial universe, $E=\left\{e_{1}, e_{2}, \ldots, e_{n}\right\}$ be a set of parameters, $\emptyset \neq V, Y, Z \subseteq E$ and $P(U)$ denotes the power set of $U$.

Definition 2.1. [2] A pair $(\Gamma, V)$ is called a s-set over $U$, where $\Gamma$ is a mapping given by $\Gamma: V \rightarrow P(U)$.

From now on, $S(U)$ denotes the family of all s-sets over $U$.

Definition 2.2. [1] Let $(\Gamma, V),(\Lambda, Y) \in S(U)$. Then, $(\Gamma, V)$ is a s-subset of $(\Lambda, Y)$ if $V \subseteq Y$ and $\Gamma(e) \subseteq \Lambda(e) ; \forall e \in V$.

We write $(\Gamma, V) \tilde{\subseteq}(\Lambda, Y)$.

Definition 2.3. [1] Let $(\Gamma, V),(\Lambda, Y) \in S(U)$. Then,

(i) the union of $(\Gamma, V)$ and $(\Lambda, Y)$ over $U$ is the s-set $(\Omega, Z)$, where $Z=V \cup Y$ and

$$
\Omega(e)= \begin{cases}\Gamma(e) & \text { if } e \in V-Y \\ \Lambda(e) & \text { if } e \in Y-V \\ \Gamma(e) \cup \Lambda(e) & \text { if } e \in V \cap Y\end{cases}
$$

for each $e \in Z$. We write $(\Gamma, V) \tilde{\cup}(\Lambda, Y)=(\Omega, Z)$.

(ii) the intersection of $(\Gamma, V)$ and $(\Lambda, Y)$ over $U$ is the s-set $(\Omega, Z)$, where $Z=V \cap Y$ and $\Omega(e)=\Gamma(e) \cap \Lambda(e) ; \forall e \in Z$. We write $(\Gamma, V) \tilde{\cap}(\Lambda, Y)=(\Omega, Z)$.

Definition 2.4. [5] Let $(\Gamma, V) \in S(U)$. Then, the complement of $(\Gamma, V)$ is a s-set $(\Gamma, V)^{c}=(\Lambda, V)$ where $\Lambda(e)=U-\Gamma(e) ; \forall e \in V$.

Definition 2.5. [1] Let $(\Gamma, E) \in S(U)$. Then, $(\Gamma, E)$ is called:

(i) A null s-set, denoted by $\tilde{\emptyset}$, if $\Gamma(e)=\emptyset ; \forall e \in E$.

(ii) An absolute s-set, denoted by $\tilde{U}$, if $\Gamma(e)=U ; \forall e \in E$.

Definition 2.6. [1] The NOT set of $E$ denoted by $\neg E$ is defined by $\neg E=$ $\left\{\neg e_{1}, \neg e_{2}, \ldots, \neg e_{n}\right\}$ where $\neg e_{i}=$ not $e_{i} ; \forall i$.

Definition 2.7. [3] A $(\Gamma, \Lambda, V)$ is called a bs-set over $U$ where $\Gamma, \Lambda$ are mappings given by $\Gamma: V \rightarrow P(U), \Lambda: \neg V \rightarrow P(U)$ such that $\Gamma(e) \cap \Lambda(\neg e)=\emptyset ; \forall e \in V$.

From now on, $B S(U)$ denotes the family of all bs-sets over $U$. 
Definition 2.8. [3] Let $(\Gamma, \Lambda, V),\left(\Gamma_{1}, \Lambda_{1}, Y\right) \in B S(U)$. Then; $(\Gamma, \Lambda, V)$ is a bssubset of $\left(\Gamma_{1}, \Lambda_{1}, Y\right)$, if

(i) $V \subseteq Y$,

(ii) $\Gamma(e) \subseteq \Gamma_{1}(e), \Lambda_{1}(\neg e) \subseteq \Lambda(\neg e) ; \forall e \in V$.

We write $(\Gamma, \Lambda, V) \tilde{\subseteq}\left(\Gamma_{1}, \Lambda_{1}, Y\right)$.

Definition 2.9. [3] Let $(\Gamma, \Lambda, V) \in B S(U)$. Then, the complement of a bs-set $(\Gamma, \Lambda, V)$ is denoted by $(\Gamma, \Lambda, V)^{c}$ and is defined by $(\Gamma, \Lambda, V)^{c}=\left(\Gamma^{c}, \Lambda^{c}, V\right)$ where $\Gamma^{c}, \Lambda^{c}$ are mappings given by $\Gamma^{c}(e)=\Lambda(\neg e), \Lambda^{c}(\neg e)=\Gamma(e) ; \forall e \in V$.

Definition 2.10. [9] Let $(\Gamma, \Lambda, V) \in B S(U)$. Then,

(i) $(\Gamma, \Lambda, V)$ is said to be relative null bs-set, denoted by $(\Phi, \tilde{U}, V)$, if $\Gamma(e)=\emptyset$; $\forall e \in V$ and $\Lambda(\neg e)=U ; \forall \neg e \in \neg V$.

(ii) the relative null bs-set with respect to $U$ of $E$ is called a NULL bs-set over $U$ and is denoted by $(\Phi, \tilde{U}, E)$.

(iii) $(\Gamma, \Lambda, V)$ over $U$ is said to be relative absolute bs-set, denoted by $(\tilde{U}, \Phi, V)$, if $\Gamma(e)=U ; \forall e \in V$ and $\Lambda(\neg e)=\emptyset ; \forall \neg e \in \neg V$.

(iv) the relative absolute bs-set with respect to $U$ of $E$ is called a ABSOLUTE bs-set over $U$ and is denoted by $(\tilde{U}, \Phi, E)$.

Definition 2.11. [3] Let $(\Gamma, \Lambda, V),\left(\Gamma_{1}, \Lambda_{1}, Y\right) \in B S(U)$. Then;

(i) extended union of $(\Gamma, \Lambda, V)$ and $\left(\Gamma_{1}, \Lambda_{1}, Y\right)$ over $U$ is the bs-set $(\Omega, \mho, Z)$ over $U$, where $Z=V \cup Y$ and

$$
\Omega(e)= \begin{cases}\Gamma(e) & \text { if } e \in V-Y \\ \Gamma_{1}(e) & \text { if } e \in Y-V \\ \Gamma(e) \cup \Gamma_{1}(e) & \text { if } e \in V \cap Y\end{cases}
$$

and

$$
\mho(\neg e)= \begin{cases}\Lambda(\neg e) & \text { if } \neg e \in(\neg V)-(\neg Y) \\ \Lambda_{1}(\neg e) & \text { if } \neg e \in(\neg Y)-(\neg V) \\ \Lambda(\neg e) \cap \Lambda_{1}(\neg e) & \text { if } \neg e \in(\neg V) \cap(\neg Y)\end{cases}
$$

for all $e \in Z$. We denote it by $(\Gamma, \Lambda, V) \tilde{\cup}\left(\Gamma_{1}, \Lambda_{1}, Y\right)=(\Omega, \mho, Z)$.

(ii) extended intersection of $(\Gamma, \Lambda, V)$ and $\left(\Gamma_{1}, \Lambda_{1}, Y\right)$ over $U$ is the bs-set $(\Omega, \mho, Z)$ over $U$, where $Z=V \cup Y$ and

$$
\Omega(e)= \begin{cases}\Gamma(e) & \text { if } e \in V-Y \\ \Gamma_{1}(e) & \text { if } e \in Y-V \\ \Gamma(e) \cap \Gamma_{1}(e) & \text { if } e \in V \cap Y\end{cases}
$$

and

$$
\mho(\neg e)= \begin{cases}\Lambda(e) & \text { if } e \in(\neg V)-(\neg Y) \\ \Lambda_{1}(e) & \text { if } e \in(\neg Y)-(\neg V) \\ \Lambda(e) \cup \Lambda_{1}(e) & \text { if } e \in(\neg V) \cap(\neg Z)\end{cases}
$$

for all $e \in Z$. We denote it by $(\Gamma, \Lambda, V) \tilde{\cap}\left(\Gamma_{1}, \Lambda_{1}, Y\right)=(\Omega, \mho, Z)$.

(iii) restricted union of $(\Gamma, \Lambda, V),\left(\Gamma_{1}, \Lambda_{1}, Y\right)$ over $U$ is the bs-set $(\Omega, \mho, Z)$, where $\emptyset \neq Z=V \cap Y$ and

$$
\Omega(e)=\Gamma(e) \cup \Lambda(e) \text { and } \mho(\neg e)=\Gamma_{1}(\neg e) \cap \Lambda_{1}(\neg e)
$$


for all $e \in Z$. We denote it by $(\Gamma, \Lambda, V) \cup_{\mathfrak{R}}\left(\Gamma_{1}, \Lambda_{1}, Y\right)=(\Omega, \mho, Z)$.

(iv) restricted intersection of $(\Gamma, \Lambda, V)$ and $\left(\Gamma_{1}, \Omega_{1}, Y\right)$ over $U$ is the bs-set $(\Omega, \mho, Z)$, where $\emptyset \neq Z=V \cap Y$ and

$$
\Omega(e)=\Gamma(e) \cap \Lambda(e) \text { and } \mho(\neg e)=\Gamma_{1}(\neg e) \cup \Lambda_{1}(\neg e)
$$

for all $e \in Z$. We denote it by $(\Gamma, \Lambda, V) \cap_{\mathfrak{R}}\left(\Gamma_{1}, \Lambda_{1}, Y\right)=(\Omega, \mho, Z)$.

Definition 2.12. [8] Let $(\Gamma, V) \in S(U)$. Then; a s-topology $\tilde{\tau}$ on $(\Gamma, V)$ is a family of s-subsets of $(\Gamma, V)$ if

(i) $(\Gamma, V), \Phi \in \tilde{\tau}$,

(ii) if $(\Omega, Z),(\Lambda, Y) \in \tilde{\tau}$, then $(\Omega, Z) \tilde{\cap}(\Lambda, Y) \in \tilde{\tau}$,

(iii) if $\left(\Gamma_{\alpha}, V_{\alpha}\right) \in \tilde{\tau}, \forall \alpha \in \wedge$; then $\left(\Gamma_{\alpha}, V_{\alpha}\right)_{\alpha \in \wedge} \in \tilde{\tau}$.

If $\tilde{\tau}$ is a s-topology on $(\Gamma, V)$, then $(\Gamma, V, \tilde{\tau})$ is called the soft topological space. Moreover; the member of $\tilde{\tau}$ is called an open s-set in $(\Gamma, V, \tilde{\tau})$. Then, $(\Gamma, V)$ is said to be closed s-set if the complement of $(\Gamma, V)$ is open s-set.

Definition 2.13. [10] Let $\tilde{\tilde{\tau}}$ be the collection of bs-sets over $U$ with $E$. If

(i) $(\Phi, \tilde{U}, E),(\tilde{U}, \Phi, E) \in \tilde{\tilde{\tau}}$,

(ii) the union of any number of bs-sets in $\tilde{\tilde{\tau}}$ belong to $\tilde{\tilde{\tau}}$,

(iii) the intersection of finite number of bs-ets in $\stackrel{\tilde{\tau}}{\text { belong to }} \tilde{\tilde{\tau}}$;

then $\tilde{\tilde{\tau}}$ is said to be a bs-topology over $U$ and $(U, \tilde{\tilde{\tau}}, E, \neg E)$ is called a bs-topological space over $U$.

Moreover; the members of $\tilde{\tau}$ are said to be bs-open sets in $U$. A bs-set $(\Gamma, \Lambda, E)$ over $X$ is said to be a bs-closed set in $X$, if its bs-complemet $(\Gamma, \Lambda, E)^{c}$ belongs to $\tilde{\tilde{\tau}}$.

Definition 2.14. $[10]$ Let $(\Gamma, \Lambda, E) \in B S(U)$ and $(X, \tilde{\tilde{\tau}}, E, \neg E)$ be a bs-topological space over $X \subseteq U$. $(\Gamma, \Lambda, E)$ over $X$ is said to be a bs-clopen set in $X$, if it is both a bs-open set and a bs-closed set over $X$.

\section{A New Definition for Bipolar Soft Topology}

Definition 3.1. Let $(\Gamma, \Lambda, E) \in B S(U)$. Then, a bs-topology $\tilde{\tilde{\tau}}$ on $(\Gamma, \Lambda, V)$ is a family of bs-subsets of $(\Gamma, \Lambda, V)$ if it satisfies the following properties

i) $(\Phi, \tilde{U}, V),(\Gamma, \Lambda, V) \in \tilde{\tilde{\tau}}$

ii) If $\left(\Gamma_{1}, \Lambda_{1}, Y\right),\left(\Gamma_{2}, \Lambda_{2}, Z\right) \in \tilde{\tilde{\tau}}$, then $\left(\Gamma_{1}, \Lambda_{1}, Y\right) \tilde{\cap}\left(\Gamma_{2}, \Lambda_{2}, Z\right) \in \tilde{\tilde{\tau}}$,

iii) If $\left(\Gamma_{\alpha}, \Lambda_{\alpha}, V_{\alpha}\right) \in \tilde{\tilde{\tau}}, \forall \alpha \in \wedge$; then $\left(\Gamma_{\alpha}, \Lambda_{\alpha}, V_{\alpha}\right)_{\alpha \in \wedge} \in \tilde{\tilde{\tau}}$.

If $\tilde{\tilde{\tau}}$ is a bs-topology on $(\Gamma, \Lambda, V)$, then $(\Gamma, \Lambda, V, \tilde{\tilde{\tau}})$ is called the bs-topological space.

Definition 3.2. Let $(\Gamma, \Lambda, E) \in B S(U)$. Then, if $\tilde{\tilde{\tau}}$ is a bs-topology on $(\Gamma, \Lambda, V)$, then the member of $\tilde{\tilde{\tau}}$ is called an open bs-set in $(\Gamma, \Lambda, V, \tilde{\tilde{\tau}})$.

Definition 3.3. Let $(\Gamma, \Lambda, V, \tilde{\tilde{\tau}})$ be a bs-topological space and $\left(\Gamma_{1}, \Lambda_{1}, Y\right) \subseteq(\Gamma, \Lambda, V)$. Then, $\left(\Gamma_{1}, \Lambda_{1}, Y\right)$ is said to be closed bs-set if the complement of $\left(\Gamma_{1}, \Lambda_{1}, Y\right)$ is open bs-set.

Definition 3.4. A collection $\tilde{\tilde{\beta}}$ of some bs-subsets of $(\Gamma, \Lambda, V)$ is called a bs-base for some bs-topology on $(\Gamma, \Lambda, V)$ if

(i) $(\Phi, \tilde{U}, V) \in \tilde{\tilde{\beta}}$, 
(ii) $\tilde{\tilde{\beta}}=(\Gamma, \Lambda, V)$ i.e. " $x \in \Gamma(e)$ and $y \in \Lambda(\neg e) ", \forall e \in V$; there exits $(K, L, Y) \in \tilde{\tilde{\beta}}$ such that $x \in K(e)$ and $y \in L(\neg e)$, where $Y \subseteq V$,

(iii) If $\left(\Gamma_{1}, \Lambda_{1}, Y\right),\left(\Gamma_{2}, \Lambda_{2}, Z\right) \in \tilde{\tilde{\beta}}$ then $x \in \Gamma_{1}(e) \cap \Gamma_{2}(e)$ and $y \in \Lambda_{1}(\neg e) \cup \Lambda_{2}(\neg e)$, $\forall e \in Y \cap Z$; there exists $\left(\Gamma_{3}, \Lambda_{3}, D\right) \subseteq \tilde{\tilde{\beta}}$ such that

$$
\left(\Gamma_{3}, \Lambda_{3}, D\right) \tilde{\subseteq}\left(\Gamma_{1}, \Lambda_{1}, Y\right) \tilde{\cap}\left(\Gamma_{2}, \Lambda_{2}, Z\right)
$$

and " $x \in \Gamma_{3}(e)$ and $y \in \Lambda_{3}(\neg e)$ ", where $D \subseteq Y \cap Z$.

Theorem 3.5. Let $(\Gamma, \Lambda, V, \tilde{\tilde{\tau}})$ be a bs-topological space. $\tilde{\tilde{\beta}}$ is a bs-base if and only if

(i) $\tilde{\tilde{\beta}} \tilde{\subseteq} \tilde{\tilde{\tau}}$,

(ii) $(M, N, Y)=\tilde{U}_{\text {for some }(K, L, Z) \in \tilde{\tilde{\beta}}}(K, L, Z) ; \forall(M, N, Y) \in \tilde{\tilde{\tau}}$.

Proof. $(\Rightarrow)(i)$ By Definition 3.4.

(ii) Let $(M, N, Y) \in \tilde{\tilde{\tau}}$. If $(M, N, Y)=(\Phi, \tilde{U}, B)$ then $(M, N, Y)=\tilde{U}_{i \in \emptyset}\left(K_{i}, L_{i}, Z_{i}\right)$. If $(M, N, Y) \neq(\Phi, \tilde{U}, Y)$ then $y \in N(\neg e), \forall x \in M(e)$; there exists a bs-set $(K, L, Z) \in \tilde{\tilde{\beta}}$ such that $(K, L, Z) \tilde{\subseteq}(M, N, Y)$ and " $x \in K(e)$ and $y \in L(\neg e)$ " where $Z \subset Y$ then $(M, N, Y)=\tilde{U}_{\text {for } \operatorname{some}(K, L, Z) \in \tilde{\tilde{\beta}}}(K, L, Z)$.

$(\Leftarrow)(i)(\Phi, U, Y)=\tilde{\bigcup}_{i \in \emptyset}\left(K_{i}, L_{i}, Z_{i}\right)$,

(ii) Since $\tilde{\tilde{\tau}}$ is a bs-topology then $(\Gamma, \Lambda, V) \in \tilde{\tilde{\tau}}$ and by $(2)(\Gamma, \Lambda, V)=\tilde{U} \tilde{\tilde{\beta}}$,

(iii) Let $\left(M_{1}, G_{1}, Y_{1}\right),\left(M_{2}, G_{2}, Y_{2}\right) \in \tilde{\tilde{\beta}}$ then $\left(M_{1}, G_{1}, Y_{1}\right),\left(M_{2}, G_{2}, Y_{2}\right) \in \tilde{\tilde{\tau}}$ since $\left(M_{1}, G_{1}, Y_{1}\right) \tilde{\cap}\left(M_{2}, G_{2}, Y_{2}\right) \in \tilde{\tilde{\tau}}$ then by $(i i)$

$$
\left(M_{1}, G_{1}, Y_{1}\right) \tilde{\cap}\left(M_{2}, G_{2}, Y_{2}\right)=\tilde{U}_{\text {for some }(K, L, Z) \in \tilde{\tilde{\beta}}}(K, L, Z) .
$$

Then for $e \in Y_{1} \cap Y_{2}$ that $(K, L, Z) \tilde{\subseteq}\left(M_{1}, G_{1}, Y_{1}\right) \tilde{\cap}\left(M_{2}, G_{2}, Y_{2}\right)$ and " $x \in K(e)$ and $y \in L(\neg e)$ " where $Z \subset Y_{1} \cap Y_{2}$.

\section{Main Results}

Definition 4.1. Let $\left(\Gamma_{1}, \Lambda_{1}, V\right),\left(\Gamma_{2}, \Lambda_{2}, Y\right) \in B S(U)$. Then, $\left(\Gamma_{1}, \Lambda_{1}, V\right) \times\left(\Gamma_{2}, \Lambda_{2}, Y\right)=$ $(\Omega, \mho, V \times Y)$ is the cartesian product of $\left(\Gamma_{1}, \Lambda_{1}, V\right)$ and $\left(\Gamma_{2}, \Lambda_{2}, Y\right)$, such that $(v, y) \in V \times Y, \Omega: V \times Y \rightarrow P(U \times U)$ and $(\neg v, \neg y) \in \neg V \times \neg Y, \mho: \neg V \times \neg Y \rightarrow$ $P(U \times U)$ where $\Omega(v, y)=\Gamma_{1}(v) \times \Gamma_{2}(y)=\left\{\left(h_{i}, h_{j}\right): h_{i} \in \Gamma_{1}(v), h_{j} \in \Gamma_{2}(y)\right\}$ and $\mho(\neg v, \neg y)=\Lambda_{1}(\neg v) \times \Lambda_{2}(\neg y)=\left\{\left(t_{i}, t_{j}\right): t_{i} \in \Lambda_{1}(\neg v), t_{j} \in \Lambda_{2}(\neg y)\right\}$.

Definition 4.2. Let $\left(\Gamma_{1}, \Lambda_{1}, V\right),\left(\Gamma_{2}, \Lambda_{2}, Y\right) \in B S(U)$. Then, a bs-set relation $\tilde{R}$ from $\left(\Gamma_{1}, \Lambda_{1}, V\right)$ to $\left(\Gamma_{2}, \Lambda_{2}, Y\right)$ is a bs-subset of $\left(\Gamma_{1}, \Lambda_{1}, V\right) \times\left(\Gamma_{2}, \Lambda_{2}, Y\right)$. In other words, a bs-set relation $\tilde{R}$ from $\left(\Gamma_{1}, \Lambda_{1}, V\right)$ to $\left(\Gamma_{2}, \Lambda_{2}, Y\right)$ is of the form $\tilde{R}=$ $\left(\Omega_{1}, \mho_{1}, S\right)$ where $S \subset V \times Y$ and $\Omega_{1}(v, y)=\Omega(v, y), \mho_{1}(\neg v, \neg y)=\mho(\neg v, \neg y)$, $\forall(v, y) \in S$ where $(\Omega, \mho, V \times Y)=\left(\Gamma_{1}, \Lambda_{1}, V\right) \times\left(\Gamma_{2}, \Lambda_{2}, Y\right)$.

Definition 4.3. Let $(\Gamma, \Lambda, V) \in B S(U)$ and $\tilde{R}$ be a bs-set relation on $(\Gamma, \Lambda, V)$, then

(1) $\tilde{R}$ is called reflexive if $\Omega_{1}(v, v) \in \tilde{R}, \mho_{1}(\neg v, \neg v) \in \tilde{R} ; \forall v \in V$.

(2) $\tilde{R}$ is called symmetric if $\Omega_{1}(v, y) \in \tilde{R} \Rightarrow \Omega_{1}(y, v) \in \tilde{R}, \mho_{1}(\neg v, \neg y) \in \tilde{R} \Rightarrow$ $\mho_{1}(\neg y, \neg v) \in \tilde{R} ; \forall v, y \in V$.

(3) $\tilde{R}$ is called transitive if $\Omega_{1}(v, y) \in \tilde{R}, \Omega_{1}(y, z) \in \tilde{R} \Rightarrow \Omega_{1}(v, z) \in \tilde{R}, \mho_{1}(\neg v, \neg z) \in$ $\tilde{R}, \mho_{1}(\neg y, \neg z) \in \tilde{R} \Rightarrow \mho_{1}(\neg v, \neg z) \in \tilde{R} ; \forall v, y, z \in V$. 
Definition 4.4. Let $(\Gamma, \Lambda, V) \in B S(U)$ and the a binary bs-set relation $\tilde{R}$ on $(\Gamma, \Lambda, V)$ is called an antisymmetric relation if $\Gamma(v) \times \Gamma(y) \in \tilde{R}, \Gamma(y) \times \Gamma(v) \in \tilde{R}$, $\Lambda(\neg v) \times \Lambda(\neg y) \in \tilde{R}$ and $\Lambda(\neg y) \times \Lambda(\neg v) \in \tilde{R}, \forall \Gamma(v), \Gamma(y) \in(\Gamma, A)$ and $\Lambda(\neg v), \Lambda(\neg y) \in$ $(\Lambda, \neg A)$ imply $\Gamma(y)=\Gamma(v)$ and $\Lambda(\neg v)=\Lambda(\neg y)$.

Definition 4.5. Let $(\Gamma, \Lambda, V) \in B S(U)$ and the a binary bs-set relation $\leq$ on $(\Gamma, \Lambda, V)$ which is reflexive, transitive and antisymmetric is called a partial ordering on a bs-set $(\Gamma, \Lambda, V)$. The quadruple $(\Gamma, \Lambda, V, \leq)$ is called a partially ordered bs-set.

Definition 4.6. Let $(\Gamma, \Lambda, V) \in B S(U)$ and $\leq$ be an ordering of $(\Gamma, \Lambda, V)$ and $(\Gamma(v), \Lambda(\neg v)),(\Gamma(y), \Lambda(\neg y))$ be any two elements in $(\Gamma, \Lambda, V)$. If " $\Gamma(v) \leq \Gamma(y)$ and $\Lambda(\neg y) \leq \Lambda(\neg v)$ " or " $\Gamma(y) \leq \Gamma(v)$ and $\Lambda(\neg v) \leq \Lambda(\neg y)$ " then $(\Gamma(v), \Lambda(\neg v))$ and $(\Gamma(y), \Lambda(\neg y))$ are comparable in the ordering. If they are not comparable, then $(\Gamma(v), \Lambda(\neg v))$ and $(\Gamma(y), \Lambda(\neg y))$ are incomparable.

Definition 4.7. Let $(\Gamma, \Lambda, Y) \in B S(U)$. Then, if $(\Gamma, \Lambda, Y, \leq)$ is a partially ordered bs-set then,

a) For $y \in Y$; if $\Gamma(y) \leq \Gamma(\beta), \Lambda(\neg \beta) \leq \Lambda(\neg y), \forall \beta \in Y$; then $(\Gamma(y), \Lambda(\neg y))$ is the least element of $(\Gamma, \Lambda, Y)$ in the ordering " $\leq$ ".

b) For $y \in Y$; if there exists no $\beta \in Y$ such that $" \Gamma(\beta) \leq \Gamma(y)$ and $\Lambda(\neg y) \leq$ $\Lambda(\neg \beta)$ " and " $\Gamma(\beta) \neq \Gamma(y)$ and $\Lambda(\neg y) \neq \Lambda(\neg \beta)$ ", then $(\Gamma(y), \Lambda(\neg y))$ is a minimal element of $(\Gamma, \Lambda, Y)$ in the ordering " $\leq "$.

$\left.a^{\prime}\right)$ For $y \in Y$; if $\Lambda(\neg y) \leq \Lambda(\neg \beta), \forall \beta \in Y \Gamma(\beta) \leq \Gamma(y)$; then $(\Gamma(y), \Lambda(\neg y))$ is the greatest element of $(\Gamma, \Lambda, Y)$ in the ordering " $\leq$.

$\left.b^{\prime}\right)$ For $y \in Y$; if there exists no $\beta \in Y$ such that $" \Gamma(y) \leq \Gamma(\beta)$ and $\Lambda(\neg \beta) \leq$ $\Lambda(\neg y)$ " and " $\Gamma(\beta) \neq \Gamma(y)$ and $\Lambda(\neg \beta) \neq \Lambda(\neg y)$ ", then $(\Gamma(y), \Lambda(\neg y))$ is a maximal element of $(\Gamma, \Lambda, Y)$ in the ordering " $\leq "$.

Definition 4.8. Let $\left(\Gamma_{1}, \Lambda_{1}, V\right),\left(\Gamma_{2}, \Lambda_{2}, Y\right) \in B S(U), \leq$ be an ordering of $\left(\Gamma_{1}, \Lambda_{1}, V\right)$ and $\left(\Gamma_{2}, \Lambda_{2}, Y\right) \subseteq\left(\Gamma_{1}, \Lambda_{1}, V\right)$.

a) For $v \in V,\left(\Gamma_{1}(v), \Lambda_{1}(\neg v)\right)$ is a lower bound of $\left(\Gamma_{2}, \Lambda_{2}, Y\right)$ in the partially ordered bs-set $\left(\Gamma_{1}, \Lambda_{1}, V, \leq\right)$ if $\Gamma_{1}(v) \leq \Gamma_{2}(\beta)$ and $\Lambda_{2}(\neg \beta) \leq \Lambda_{1}(\neg v) ; \forall \beta \in Y$.

b) For $v \in V,\left(\Gamma_{1}(v), \Lambda_{1}(\neg v)\right)$ is called infimum of $\left(\Gamma_{2}, \Lambda_{2}, Y\right)$ in $\left(\Gamma_{1}, \Lambda_{1}, V, \leq\right)$ if it is the greatest element of the set of all lower bounds of the bs-subset $\left(\Gamma_{2}, \Lambda_{2}, Y\right)$ in $\left(\Gamma_{1}, \Lambda_{1}, V, \leq\right)$.

Similarly,

$\left.a^{\prime}\right)$ For $v \in V,\left(\Gamma_{1}(v), \Lambda_{1}(\neg v)\right)$ is an upper bound of $\left(\Gamma_{2}, \Lambda_{2}, Y\right)$ in the partially ordered bs-set $\left(\Gamma_{1}, \Lambda_{1}, V, \leq\right)$ if $\Gamma_{2}(\beta) \leq \Gamma_{1}(v), \Lambda_{1}(\neg v) \leq \Lambda_{2}(\neg \beta) ; \forall \beta \in Y$.

$\left.b^{\prime}\right)$ For $v \in V,\left(\Gamma_{1}(v), \Lambda_{1}(\neg v)\right)$ is called supremum of $\left(\Gamma_{2}, \Lambda_{2}, Y\right)$ in $\left(\Gamma_{1}, \Lambda_{1}, V, \leq\right)$ if it is the least element of the set of all upper bounds of the s-subset $\left(\Gamma_{2}, \Lambda_{2}, Y\right)$ in $\left(\Gamma_{1}, \Lambda_{1}, V, \leq\right)$. 


\subsection{Bipolar Soft Intervals.}

Definition 4.9. Let $(\Gamma, \Lambda, V) \in B S(U)$ and $\tilde{R}$ be a bs-set relation on $(\Gamma, \Lambda, V)$. If for no $v \in V, \Gamma(v)$ and $\Lambda(\neg v)$ the s-set relation $\Gamma(v) \tilde{R} \Gamma(v)$ and $\Lambda(\neg v) \tilde{R} \Lambda(\neg v)$ hold, the bs-set relation $\tilde{R}$ is called nonreflexive.

Definition 4.10. Let $(\Gamma, \Lambda, V) \in B S(U)$ and a bs-set relation $\tilde{R}$ on $(\Gamma, \Lambda, V)$ is called simple order bs-set relation if it is comparable, nonreflexive and transitive. $(\Gamma, \Lambda, V)$ is called a simple ordered bs-set a the simple order bs-set relation $\tilde{R}$.

Definition 4.11. Let $\leq$ be a bs-set relation on $(\Gamma, \Lambda, V)$, then restriction of a bsset relation $\leq$ to a bs-subset $\left(\Gamma_{1}, \Lambda_{1}, Y\right)$ is defined as follows:

We denote $\Gamma_{1}(v) \leq_{\left(\Gamma_{1}, \Lambda_{1}, Y\right)} \Gamma_{1}(y)$ and $\Lambda_{1}(\neg y) \leq_{\left(\Gamma_{1}, \Lambda_{1}, Y\right)} \Lambda_{1}(\neg v)$ : if and only if $\Gamma(v) \leq \Gamma(y)$ and $\Lambda(\neg y) \leq \Lambda(\neg v) ; \forall v, y \in B$.

Example 4.12. Let $V=\left\{m_{1}, m_{2}, m_{3}\right\}$ be a parameter set and $U=\left\{u_{1}, u_{2}, u_{3}, u_{4}, u_{5}, u_{6}\right\}$ be a universe set. $\Gamma\left(m_{1}\right)=\left\{u_{1}\right\}, \Gamma\left(m_{2}\right)=\left\{u_{2}, u_{4}, u_{5}\right\}, \Gamma\left(m_{3}\right)=\left\{u_{3}\right\} \Lambda\left(\neg m_{1}\right)=$ $\left\{u_{3}, u_{4}\right\}, \Lambda\left(\neg m_{2}\right)=\left\{u_{1}, u_{5}\right\}, \Lambda\left(\neg m_{3}\right)=\left\{u_{2}\right\} ; Y=\left\{m_{1}, m_{2}\right\}, \Gamma_{1}\left(m_{1}\right)=\left\{u_{1}\right\}$, $\Gamma_{1}\left(m_{2}\right)=\left\{u_{2}, u_{4}\right\}, \Lambda_{1}\left(\neg m_{1}\right)=\left\{u_{4}\right\}, \Lambda_{1}\left(\neg m_{2}\right)=\left\{u_{1}\right\}$. Then $\left(\Gamma_{1}, \Lambda_{1}, Y\right) \subseteq$ $(\Gamma, \Lambda, V)$.

$$
\begin{aligned}
& \leq_{(\Gamma, \Lambda, V)}=\left\{\begin{array}{c}
\Gamma\left(m_{1}\right) \times \Gamma\left(m_{2}\right), \Gamma\left(m_{2}\right) \times \Gamma\left(m_{3}\right), \\
\Lambda\left(\neg m_{1}\right) \times \Lambda\left(\neg m_{2}\right), \Lambda\left(\neg m_{2}\right) \times \Lambda\left(\neg m_{3}\right)
\end{array}\right\} \\
&=\left\{\begin{array}{c}
\left(u_{1}, u_{2}\right),\left(u_{1}, u_{4}\right),\left(u_{1}, u_{5}\right),\left(u_{2}, u_{3}\right),\left(u_{4}, u_{3}\right),\left(u_{5}, u_{3}\right), \\
\left(u_{3}, u_{1}\right),\left(u_{3}, u_{5}\right),\left(u_{4}, u_{1}\right),\left(u_{4}, u_{5}\right),\left(u_{1}, u_{2}\right),\left(u_{5}, u_{2}\right)
\end{array}\right\} \\
& \text { Then } \leq_{\left(\Gamma_{1}, \Lambda_{1}, Y\right)}=\left\{\begin{array}{c}
\Gamma_{1}\left(m_{1}\right) \times \Gamma_{1}\left(m_{2}\right), \\
\Lambda_{1}\left(\neg m_{1}\right) \times \Lambda_{1}\left(\neg m_{2}\right)
\end{array}\right\}=\left\{\left(u_{1}, u_{2}\right),\left(u_{1}, u_{4}\right),\left(u_{4}, u_{1}\right)\right\} .
\end{aligned}
$$

Definition 4.13. Assume that $(\Gamma, \Lambda, V)$ is a bs-set having a simple order bs-set relation $<$ and $(\Gamma(v), \Lambda(\neg v))$ and $(\Gamma(y), \Lambda(\neg y))$ be elements of $(\Gamma, \Lambda, V)$ such that $\Gamma(v)<\Gamma(y)$ and $\Lambda(\neg y)<\Lambda(\neg v)$. Then we can define following four bs-subsets of $(\Gamma, \Lambda, V)$ which are called bs-intervals (respectively; bs-closed interval, bs-half open intervals, bs-open interval) determined by $(\Gamma(v), \Lambda(\neg v))$ and $(\Gamma(y), \Lambda(\neg y))$ :

a) bs-Open Interval: The bs-open interval is a bs-subset $\left(\Gamma_{1}, \Lambda_{1}, Y\right)$ of $(\Gamma, \Lambda, V)$ where " $Y_{1}=\{x: \Gamma(v)<\Gamma(x)<\Gamma(y)\}$ and $Y_{2}=\{\neg x: \Lambda(\neg y)<\Lambda(\neg x)<\Lambda(\neg v)\} "$, $" \Gamma_{1}=\left.\Gamma\right|_{Y}$ and $\Lambda_{1}=\left.\Lambda\right|_{Y}$ " and denoted by $(\Gamma(v), \Gamma(y))=\{\Gamma(x): \Gamma(v)<\Gamma(x)<$ $\Gamma(y)\}$ and $(\Lambda(\neg y), \Lambda(\neg v))=\{\Lambda(\neg x): \Lambda(\neg y)<\Lambda(\neg x)<\Lambda(\neg v)\}$.

\section{b) bs-Half Open Interval:}

(i) The bs-open interval is a bs-subset $\left(\Gamma_{1}, \Lambda_{1}, Y\right)$ of $(\Gamma, \Lambda, V)$ where $B_{1}=$ $\{x: \Gamma(v)<\Gamma(x)<\Gamma(y)$ or $\Gamma(x)=\Gamma(y)\}$ and $Y_{2}=\{\neg x: \Lambda(\neg y)<\Lambda(\neg x)<$ $\Lambda(\neg a)$ or $\Lambda(\neg x)=\Lambda(\neg y)\}$, " $\Gamma_{1}=\left.\Gamma\right|_{Y}$ and $\Lambda_{1}=\left.\Lambda\right|_{Y}$ " denoted by $(\Gamma(v), \Gamma(y)]=$ $\{\Gamma(x): \Gamma(v)<\Gamma(x)<\Gamma(y)$ or $\Gamma(x)=\Gamma(y)\}$ and $(\Lambda(\neg y), \Lambda(\neg v)]=\{\Lambda(\neg x)$ : $\Lambda(\neg y)<\Lambda(\neg x)<\Lambda(\neg v)$ or $\Lambda(\neg x)=\Lambda(\neg y)\}$.

(ii) The bs-open interval is a bs-subset $\left(\Gamma_{1}, \Lambda_{1}, Y\right)$ of $(\Gamma, \Lambda, V)$ where $Y_{1}=$ $\{x: \Gamma(v)<\Gamma(x)<\Gamma(y)$ or $\Gamma(x)=\Gamma(v)\}$ and $Y_{2}=\{\neg x: \Lambda(\neg y)<\Lambda(\neg x)<$ $\Lambda(\neg v)$ or $\Lambda(\neg x)=\Lambda(\neg v)\}, " \Gamma_{1}=\left.\Gamma\right|_{Y}$ and $\Lambda_{1}=\left.\Lambda\right|_{Y}$ " and denoted by $[\Gamma(v), \Gamma(y))=$ 
$\{\Gamma(x): \Gamma(v)<\Gamma(x)<\Gamma(y)$ or $\Gamma(x)=\Gamma(v)\}$ and $[\Lambda(\neg y), \Lambda(\neg v))=\{\Lambda(\neg x):$ $\Lambda(\neg y)<\Lambda(\neg x)<\Lambda(\neg v)$ or $\Lambda(\neg x)=\Lambda(\neg v)\}$.

d) bs-Closed Interval: The bs-open interval is a bs-subset $\left(\Gamma_{1}, \Lambda_{1}, Y\right)$ of $(\Gamma, \Lambda, V)$ where $Y_{1}=\{x: \Gamma(v)<\Gamma(x)<\Gamma(y)$ or $\Gamma(x)=\Gamma(v)$ or $\Gamma(x)=\Gamma(y)\}$ and $Y_{2}=\{\neg x: \Lambda(\neg y)<\Lambda(\neg x)<\Lambda(\neg v)$ or $\Lambda(\neg x)=\Lambda(\neg y)$ or $\Lambda(\neg x)=\Lambda(\neg v)\}$, $" \Gamma_{1}=\left.\Gamma\right|_{Y}$ and $\Lambda_{1}=\left.\Lambda\right|_{Y}$ " and denoted by $[\Gamma(v), \Gamma(y)]=\{\Gamma(x): \Gamma(v)<\Gamma(x)<$ $\Gamma(y)$ or $\Gamma(x)=\Gamma(v)$ or $\Gamma(x)=\Gamma(y)\}$ and $[\Lambda(\neg y), \Lambda(\neg v)]=\{\Lambda(\neg x): \Lambda(\neg y)<$ $\Lambda(\neg x)<\Lambda(\neg v)$ or $\Lambda(\neg x)=\Lambda(\neg v)$ or $\Lambda(\neg x)=\Lambda(\neg y)\}$.

These are the bs-intervals on an arbitrary simple ordered bs-set.

Example 4.14. Let $V=\left\{v_{1}, v_{2}, v_{3}, v_{4}, v_{5}\right\}$ be the parameter set and $U=\left\{m_{1}, m_{2}, m_{3}, m_{4}, m_{5}, m_{6}\right\}$ be the universe set. Lets define a bs-set $(\Gamma, \Lambda, V)$ such that $\Gamma\left(v_{1}\right)=\left\{m_{1}, m_{4}\right\}$, $\Gamma\left(v_{2}\right)=\left\{m_{1}, m_{2}, m_{6}\right\}, \Gamma\left(v_{3}\right)=\left\{m_{2}, m_{3}, m_{5}\right\}, \Gamma\left(v_{4}\right)=\left\{m_{2}, m_{3}\right\}, \Gamma\left(v_{5}\right)=\left\{m_{1}\right\}$, $\Lambda\left(\neg v_{1}\right)=\left\{m_{2}, m_{3}\right\}, \Lambda\left(\neg v_{2}\right)=\left\{m_{3}, m_{4}, m_{5}\right\}, \Lambda\left(\neg v_{3}\right)=\left\{m_{1}, m_{4}, m_{6}\right\}, \Lambda\left(\neg v_{4}\right)=$ $\left\{m_{1}, m_{4}\right\}, \Lambda\left(\neg v_{5}\right)=\left\{m_{2}\right\}$. Consider a bs-set relation on $(\Gamma, \Lambda, V)$ defined by

$$
<_{(\Gamma, \Lambda, V)}=\left\{\begin{array}{c}
\Gamma\left(v_{2}\right) \times \Gamma\left(v_{3}\right), \Gamma\left(v_{1}\right) \times \Gamma\left(v_{2}\right), \Gamma\left(v_{2}\right) \times \Gamma\left(v_{4}\right), \\
\Gamma\left(v_{2}\right) \times \Gamma\left(v_{5}\right), \Gamma\left(v_{1}\right) \times \Gamma\left(v_{3}\right), \Gamma\left(v_{3}\right) \times \Gamma\left(v_{4}\right), \\
\Gamma\left(v_{3}\right) \times \Gamma\left(v_{5}\right), \Gamma\left(v_{1}\right) \times \Gamma\left(a_{4}\right), \Gamma\left(v_{1}\right) \times \Gamma\left(v_{5}\right), \\
\Gamma\left(v_{4}\right) \times \Gamma\left(v_{5}\right), \Lambda\left(\neg v_{3}\right) \times \Lambda\left(\neg v_{2}\right), \Lambda\left(\neg v_{2}\right) \times \Lambda\left(\neg v_{1}\right), \\
\Lambda\left(\neg v_{4}\right) \times \Lambda\left(\neg v_{2}\right), \Lambda\left(\neg v_{5}\right) \times \Lambda\left(\neg v_{2}\right), \Lambda\left(\neg v_{3}\right) \times \Lambda\left(\neg v_{1}\right), \\
\Lambda\left(\neg v_{4}\right) \times \Lambda\left(\neg v_{3}\right), \Lambda\left(\neg v_{5}\right) \times \Lambda\left(\neg v_{3}\right), \Lambda\left(\neg v_{4}\right) \times \Lambda\left(\neg v_{1}\right), \\
\Lambda\left(\neg v_{5}\right) \times \Lambda\left(\neg v_{1}\right), \Lambda\left(\neg v_{5}\right) \times \Lambda\left(\neg v_{4}\right)
\end{array}\right\} .
$$

The bs-set relation " $<$ " is comparable, nonreflexive, transitive so it is simple ordered bs-set relation.

Definition 4.15. Let $(\Gamma, \Lambda, V)$ be an simple ordered bs-set with a bs-set relation $<$ and $(\Gamma(v), \Lambda(\neg v))$ be in $(\Gamma, \Lambda, V)$. Then there are four soft subsets of $(\Gamma, \Lambda, V)$ which are called bs-rays determined $(\Gamma(a), \Lambda(\neg v))$. They are following:

i) $((\Gamma(v), *) \cup(*, \Lambda(\neg v))=\{(\Gamma(x), \Lambda(\neg x)): \Gamma(v)<\Gamma(x)$ and $\Lambda(\neg x)<\Lambda(\neg v)\}$ is called bs-open ray,

ii) $(*, \Gamma(v)) \cup(\Lambda(\neg v), *)=\{(\Gamma(x), \Lambda(\neg x)): \Gamma(x)<\Gamma(v)$ and $\Lambda(\neg v)<\Lambda(\neg x)\}$ is called bs-open ray,

iii $)[\Gamma(v), *) \cup(\Lambda(\neg v), *]=\left\{\begin{array}{cc}{[\Gamma(x), \Lambda(\neg x)):} & \left.\begin{array}{c}\Gamma(v)<\Gamma(x) \\ \text { or } \\ \Gamma(v)=\Gamma(x)\end{array}\right] \\ \text { and } \\ & {\left[\begin{array}{c}\Lambda(\neg x)<\Lambda(\neg v) \\ \text { or } \\ \Lambda(\neg x)=\Lambda(\neg v)\end{array}\right]}\end{array}\right\}$ is called bs-

closed ray, 
$i v)(\Gamma(v), *] \cup[\Lambda(\neg v), *)=\left\{(\Gamma(x), \Lambda(\neg x)): \quad \begin{array}{c}\Gamma(x)<\Gamma(v) \\ \text { or } \\ \Gamma(x)=\Gamma(v)\end{array}\right]$ and $\left.\begin{array}{c}\Lambda(\neg v)<\Lambda(\neg x) \\ \text { or } \\ \Lambda(\neg v)=\Lambda(\neg x)\end{array}\right]$ is called bsclosed ray.

\subsection{Bipolar Soft Ordered Topology.}

Theorem 4.16. Let $(\Gamma, \Lambda, V)$ be a bs-set with a simple ordered bs-set relation; assume that $(\Gamma, \Lambda, V)$ has more then one element. Let $\tilde{\tilde{\beta}}$ be a collection of all bssubsets of $(\Gamma, \Lambda, V)$ of the following types:

(1) $(\Phi, \tilde{U}, V)$,

(2) All bs-open intervals $(\Gamma(v), \Gamma(y))$ and $(\Lambda(\neg y), \Lambda(\neg v))$ in $(\Gamma, \Lambda, V)$,

(3) All bs-intervals of from $\left[\Gamma\left(v_{0}\right), \Gamma(y)\right)$ and $\left(\Lambda(\neg y), \Lambda\left(\neg v_{0}\right)\right]$, where $\Gamma\left(v_{0}\right)$ is the least element of $(\Gamma, V)$ and $\Lambda\left(\neg v_{0}\right)$ is the greatest element of $(\Lambda, \neg V)$,

(4) All bs-intervals of the form $\left(\Gamma(v), \Gamma\left(y_{0}\right)\right]$ and $\left[\Lambda\left(\neg y_{0}\right), \Lambda(\neg v)\right)$, where $\Gamma\left(y_{0}\right)$ is the greatest element of $(\Gamma, V)$ and $\Lambda\left(y_{0}\right)$ is the least element of $(\Lambda, \neg V)$.

Then the collection $\tilde{\tilde{\beta}}$ is a bs-base for a bs-topology on $(\Gamma, \Lambda, V)$.

If $(\Gamma, V)$ has no least element or $(\Lambda, \neg V)$ has no greatest element, there is no $s$-sets in type (3). Moreover; if $(\Gamma, V)$ has no greatest element or $(\Lambda, \neg V)$ has no least element, there is no s-sets in type (4).

Proof. Lets check $\tilde{\tilde{\beta}}$ satisfies the requirements for being a bs-base given in the Definition 3.4.

(1) $(\Phi, \tilde{U}, V) \in \tilde{\tilde{\beta}}$

(2) Take $e \in V, x \in \Gamma(e)$ and $y \in \Lambda(\neg e)$. By comparability there exists a bs-interval $\left(\Gamma_{1}, \Lambda_{1}, B\right)$, where $x \in Y \subset V$ and $x \in \Gamma_{1}(e)$ and $y \in \Lambda_{1}(\neg e)$.

(3) Let $\left(\Gamma_{1}, G_{1}, Y\right),\left(\Gamma_{2}, \Lambda_{2}, Z\right) \in \tilde{\tilde{\beta}}$, where $\left(\Gamma_{1}, \Lambda_{1}, Y\right)=\left(\begin{array}{c}(\Gamma(v), \Gamma(y)), \\ (\Lambda(\neg y), \Lambda(\neg v))\end{array}\right)$, $\left(\Gamma_{2}, \Lambda_{2}, Z\right)=\left(\begin{array}{c}(\Gamma(z), \Gamma(d)), \\ (\Lambda(\neg d), \Lambda(\neg z))\end{array}\right)$. Then

$\left(\Gamma_{1}, G_{1}, Y\right) \tilde{\cap}\left(\Gamma_{2}, \Lambda_{2}, Z\right)=\left(\begin{array}{c}(\Gamma(v), \Gamma(y)), \\ (\Lambda(\neg y), \Lambda(\neg v))\end{array}\right) \tilde{\cap}\left(\begin{array}{c}(\Gamma(z), \Gamma(d)), \\ (\Lambda(\neg d), \Lambda(\neg z))\end{array}\right)=$

$\begin{cases}(\Phi, \tilde{U}, V) & (\Gamma(y)<\Gamma(z)) \vee(\Lambda(\neg z)<\Lambda(\neg y)) \\ (\Phi, \tilde{U}, V) & (\Gamma(d)<\Gamma(v)) \vee(\Lambda(\neg v)<\Lambda(\neg d)) \\ (\Gamma(z), \Gamma(y)) \wedge(\Lambda(\neg y), \Lambda(\neg z)) & (\Gamma(z)<\Gamma(y)) \vee(\Lambda(\neg y)<\Lambda(\neg z)) \\ (\Gamma(v), \Gamma(d)) \wedge(\Lambda(\neg d), \Lambda(\neg v)) & (\Gamma(v)<\Gamma(d)) \vee(\Lambda(\neg d)<\Lambda(\neg v)) \\ (\Gamma(z), \Gamma(d)) \wedge(\Lambda(\neg d), \Lambda(\neg z)) & ((\Gamma(v)<\Gamma(z)) \wedge(\Gamma(y)<\Gamma(d))) \\ (\Gamma(z), \Gamma(d)) \wedge(\Lambda(\neg d), \Lambda(\neg z)) & ((\Lambda(\neg z)<\Lambda(\neg v)) \wedge(\Lambda(\neg d)<\Lambda(\neg y))) \\ (\Gamma(v), \Gamma(d)) \wedge(\Lambda(\neg d), \Lambda(\neg v)) & ((\Gamma(z)<\Gamma(v)) \wedge(\Gamma(d)<\Gamma(y))) \\ (\Gamma(v), \Gamma(d)) \wedge(\Lambda(\neg d), \Lambda(\neg v)) & ((\Lambda(\neg v)<\Lambda(\neg z)) \wedge(\Lambda(\neg y)<\Lambda(\neg d))) \\ \left(\Gamma\left(v^{\prime}\right), \Gamma\left(d^{\prime}\right)\right) \wedge\left(\Lambda\left(\neg d^{\prime}\right), \Lambda\left(\neg v^{\prime}\right)\right) & \end{cases}$


Example 4.17. Let $V=\left\{v_{1}, v_{2}, v_{3}\right\}$ be a parameter set and $U=\left\{u_{1}, u_{2}, u_{3}, u_{4}, u_{5}\right\}$ be a universe set. $K\left(v_{1}\right)=\left\{u_{1}, u_{2}\right\}, K\left(v_{2}\right)=\left\{u_{2}\right\}, K\left(v_{3}\right)=\left\{u_{3}, u_{4}, u_{5}\right\}$;

$$
<={ }_{(K, L, M)}=\left\{\begin{array}{c}
K\left(v_{1}\right) \times K\left(v_{2}\right), K\left(v_{2}\right) \times K\left(v_{3}\right), K\left(v_{1}\right) \times K\left(v_{3}\right), \\
L\left(\neg v_{2}\right) \times L\left(v_{1}\right), L\left(\neg v_{3}\right) \times L\left(\neg v_{2}\right), L\left(\neg v_{3}\right) \times L\left(\neg v_{1}\right)
\end{array}\right\} .
$$

Then the bs-ordered topology is;

$$
\tilde{\tilde{\tau}}=\left\{\begin{array}{c}
(\Phi, \tilde{U}, A),\left[\Gamma\left(v_{1}\right), \Gamma\left(v_{2}\right)\right),\left(\Gamma\left(v_{1}\right), \Gamma\left(v_{2}\right)\right),\left[\Gamma\left(v_{1}\right), \Gamma\left(v_{3}\right)\right), \\
\left(\Gamma\left(v_{1}\right), \Gamma\left(v_{3}\right)\right),\left[\Gamma\left(v_{1}\right), \Gamma\left(v_{3}\right)\right],\left(\Gamma\left(v_{2}\right), \Gamma\left(v_{3}\right)\right),\left(\Gamma\left(v_{2}\right), \Gamma\left(v_{3}\right)\right], \\
\left(\Lambda\left(\neg v_{2}\right), \Lambda\left(\neg v_{1}\right)\right],\left(\Lambda\left(\neg v_{2}\right), \Lambda\left(\neg v_{1}\right)\right),\left(\Lambda\left(\neg v_{3}\right), \Lambda\left(\neg v_{1}\right)\right], \\
\left(\Lambda\left(\neg v_{3}\right), \Lambda\left(\neg v_{1}\right)\right),\left[\Lambda\left(\neg v_{3}\right), \Lambda\left(\neg v_{1}\right)\right], \\
\left(\Lambda\left(\neg v_{3}\right), \Lambda\left(\neg v_{2}\right)\right),\left[\Lambda\left(\neg v_{3}\right), \Lambda\left(\neg v_{2}\right)\right)
\end{array}\right\} .
$$

Example 4.18. Let $U=\mathbb{R}, V=\left[\frac{3}{2}, \infty\right), \neg V=\left(\neg \infty, \neg \frac{3}{2}\right]$ and $(\Gamma, \Lambda, V)$ be a bs-set where $\Gamma(v)=\left(\frac{3}{2}, v\right]$ and $\Lambda(\neg v)=\left(\neg v, \neg-\frac{3}{2}\right] ; \forall v \in V$. Lets define simple ordered on $(\Gamma, \Lambda, V)$ as follows: $\Gamma(v)<\Gamma(y): \Leftrightarrow v<y: \Leftrightarrow\left(\frac{3}{2}, v\right] \subseteq\left(\frac{3}{2}, y\right]$ and $\Lambda(\neg y)<\Lambda(\neg v): \Leftrightarrow \neg y<\neg v: \Leftrightarrow\left(\neg v, \neg-\frac{3}{2}\right] \subseteq\left(\neg y, \neg-\frac{3}{2}\right] . \Gamma\left(\frac{3}{2}\right)$ is the smallest element and,$\Lambda\left(\neg-\frac{3}{2}\right)$ is the biggest element, so

$$
\tilde{\tilde{\beta}}=\left\{\begin{array}{cc}
{\left[\Gamma\left(\frac{3}{2}\right), \Gamma(v)\right),\left[\Lambda(\neg v), \Lambda\left(\neg-\frac{3}{2}\right)\right),} & \Gamma(v), \Gamma(y) \in(\Gamma, V) \\
(\Gamma(v), \Gamma(y)),(\Lambda(\neg v), \Lambda(\neg y)) & \Lambda(\neg v), \Lambda(\neg y) \in(\Lambda, \neg V)
\end{array}\right\}
$$

is a bs-base for the bs-ordered topology on $(\Gamma, \Lambda, V)$.

Example 4.19. Let $U=(-\infty,-1] \cup[1, \infty)$ be the initial universe and $V=Z^{-}$ be the parameter set and $\neg V=Z^{+}$, let $(\Gamma, \Lambda, V)$ be a bs-set, defined by $(\Gamma, V)=$ $\{\Gamma(v)=(v,-1]: v \in V\}$ and $(\Lambda, V)=\{\Lambda(\neg v)=(\neg v, \neg 1]: \neg v \in \neg V\}$. Consider the bs-set relation $<$ on $(\Gamma, \Lambda, V)$, which is defined by $\Gamma(v)<\Gamma(y): \Leftrightarrow v<y$ and $\Lambda(\neg y)<\Lambda(\neg v): \Leftrightarrow y<v$. $(\Gamma, \Lambda, V)$ is a simple ordered bs-set with the relation $<$. By examining the bs-subsets of $(\Gamma, \Lambda, V), F(-1)$ is the biggest element and $\Lambda(\neg 1)$ is the smallest element . $\tilde{\tilde{\beta}}=\{(\Gamma(v), \Gamma(-1)],(\Gamma(v), \Gamma(y)),[\Lambda(\neg 1), \Lambda(\neg v)),(\Lambda(\neg y), \Lambda(\neg v))$ : $\Gamma(v), \Gamma(y) \in(\Gamma, V)$ and $\Lambda(\neg v), \Lambda(\neg y) \in(\Lambda, \neg V)\}$ is a base for the bs-ordered topology.

Definition 4.20. Let $(\Gamma, \Lambda, V, \tilde{\tilde{\tau}})$ be a bs-topological space and $\tilde{\mathcal{B S}}$ be a collection of nonnull bs-open subsets of $(\Gamma, \Lambda, V)$. If finite intersection of the elements of $\tilde{\mathcal{B S}}$ is a base for $\tilde{\tilde{\tau}}$ then $\tilde{\mathcal{B}} \tilde{\mathcal{S}}$ is called bs-subbase, ie.;

$$
\tilde{\mathcal{Y}}_{\tilde{\mathcal{B S}}}=\left\{\tilde{\cap}_{j \in J}\left(Y_{j}, S_{j}, V_{j}\right): J \text { is a finite and for all } j \in J,\left(Y_{j}, S_{j}, V_{j}\right) \in \tilde{\mathcal{B S}}\right\}
$$

Theorem 4.21. Let $(\Gamma, \Lambda, V)$ be a nonnull bs-set and $\tilde{\mathcal{B S}}$ be a collection of bssubsets of $(\Gamma, \Lambda, V)$. Then there exists a bs-topology on $(\Gamma, \Lambda, V)$ which has as a subbase $\tilde{\mathcal{B S}}$.

Proof. Lets show that $\tilde{\mathcal{Y}}_{\mathcal{B S S}}=\left\{\tilde{\cap}_{j \in J}\left(Y_{j}, S_{j}, V_{j}\right): J\right.$ is a finite and for all $j \in$ $\left.J,\left(Y_{j}, S_{j} V_{j}\right) \in \tilde{\mathcal{B S}}\right\}$ satisfies the conditions of being a bs-base

(1) $(\Gamma, \Lambda, V)=\tilde{\cap}_{j \in J}\left(Y_{j}, S_{j}, V_{j}\right)$ then $(\Gamma, \Lambda, V) \in \tilde{\mathcal{Y}}_{\mathcal{B} \mathcal{S}}$.

(2) Let $\left(\Gamma_{1}, \Lambda_{1}, Y\right),\left(\Gamma_{2}, \Lambda_{2}, B\right) \in \tilde{\mathcal{Y}}_{\tilde{\mathcal{B} S}} \Rightarrow$ If $\left(\Gamma_{1}, \Lambda_{1}, Y\right) \tilde{\cap}\left(\Gamma_{2}, \Lambda_{2}, Y\right)=(\Phi, \tilde{U}, Y) \Rightarrow$ $\left(\Gamma_{1}, v_{1}, Y\right) \tilde{\cap}\left(\Gamma_{2}, \Lambda_{2}, Y\right)=\tilde{\cup}_{j \in \emptyset}\left(\Gamma_{j}, \Lambda_{j}, Y_{j}\right)$. 


$$
\begin{aligned}
& \text { If }\left(\Gamma_{1}, \Lambda_{1}, Y\right) \tilde{\cap}\left(\Gamma_{2}, \Lambda_{2}, Y\right) \neq(\Phi, U, Y) \Rightarrow \text { since }\left(\Gamma_{1}, \Lambda_{1}, Y\right)=\tilde{\cup}_{i=1}^{n}\left(Y_{i}, S_{i}, V_{i}\right), \\
& \left(\Gamma_{2}, \Lambda_{2}, Y\right)=\tilde{\cup}_{j=1}^{m}\left(Y_{j}, S_{j}, V_{j}\right), \\
& \left(\Gamma_{1}, \Lambda_{1}, Y\right) \tilde{\cap}\left(\Gamma_{2}, \Lambda_{2}, Y\right)=\tilde{\cup}_{i=1}^{n}\left(Y_{i}, S_{i}, V_{i}\right) \tilde{\cap} \tilde{\cup}_{j=1}^{m}\left(Y_{j}, S_{j}, V_{j}\right)=\tilde{\cup}_{j=1}^{m}\left(Y_{j}, S_{j}, V_{j}\right) .
\end{aligned}
$$

This is finite intersection of elements of $\tilde{\mathcal{B S}}$ so in $\tilde{\mathcal{Y}}_{\tilde{\mathcal{B S}}}$. Therefore $\tilde{\mathcal{Y}}_{\tilde{\mathcal{B S}}}$ is a bsbase.

\section{Conclusion}

The aim of this study is to give some applications by defining the concept of bipolar soft ordered topology and to lead the studies that can be done on this bipolar soft ordered topological structure. For this, we first gave a new concept for the bipolar soft topology. We also established a relationship $\tilde{R}$ on a bipolar soft set by completing the concept of bipolar soft interval. Finally, thanks to $\tilde{R}$, the concept of bipolar soft ordered topology and some examples on this bipolar soft ordered topology are given.

\section{Acknowledgments}

The authors would like to thank the reviewers and editors of Journal of Universal Mathematics.

\section{Funding}

The author(s) declared that has no received any financial support for the research, authorship or publication of this study.

\section{The Declaration of Conflict of Interest/ Common Interest}

The author(s) declared that no conflict of interest or common interest

\section{The Declaration of Ethics Committee Approval}

This study does not be necessary ethical committee permission or any special permission.

\section{The Declaration of Research and Publication Ethics}

The author(s) declared that they comply with the scientific, ethical, and citation rules of Journal of Universal Mathematics in all processes of the study and that they do not make any falsification on the data collected. Besides, the author(s) declared that Journal of Universal Mathematics and its editorial board have no responsibility for any ethical violations that may be encountered and this study has not been evaluated in any academic publication environment other than Journal of Universal Mathematics.

\section{REFERENCES}

[1] P.K. Maji, R. Biswas, A.R. Roy, Soft set theory, Computers and Mathematics with Applications, Vol.45 No.4-5, pp.555-562, (2003).

[2] D. Molodtsov, Soft set theory first results, Comput. Math. Appl., Vol.37, No.19-31, (1999).

[3] M. Shabir, M. Naz, On Bipolar Soft Sets, arXiv: 1303.1344v1 [math.LO], (2013).

[4] K.V. Babitha, J.J. Sunil, Soft Set Relations and Functions, Comput. Math. Appl., Vol.60, pp.1840-1849, (2010). 
[5] N. Çağman, S. Karataş, S. Enginoglu, Soft topology, Comput. Math. Appl., Vol.62, pp.351-258, (2011).

[6] K.V. Babitha, J.J. Sunil, Transitive Closures and Ordering on Soft Sets, Comput. Math. Appl., Vol.62, pp.2235-2239, (2011).

[7] B. Tanay, G. Yaylalı, New structures On Partially Ordered Soft Sets and Soft Scott Topology, Ann. Fuzzy Math. Inform., Vol.7, pp.89-97, (2014).

[8] S. Roy, T.K. Samanta, An Introduction of a Soft Topological Spaces Proceeding of UGC sponsored National seminar on Recent trends in Fuzzy set theory, Rough set theory and Soft set theory at Uluberia College on 23rd and 24th September, ISBN 978-81-922305-5-9, pp.9-12, (2011).

[9] M. Shabir, M. Naz, On bipolar soft sets, Retrieved from https://arxiv.org/abs/1303.1344, (2013).

[10] M. Shabir, A. Bakhtawar, Bipolar soft connected, bipolar soft disconnected and bipolar soft compact spaces, Songklanakari J. Sci. Technol., Vol.39, No.3, pp.359-371, (2017).

[11] X. Guan, Y. Li, F. Feng, A new order relation on fuzzy soft sets and its applications, Soft Compt., Vol.17, pp.63-70, (2013).

[12] I.A. Onyeozili, T.M. Gwary, A study the Fundamentals of Soft Set Theory, International of Sciences and Technology Research, Vol.3, No.4, pp.132-143, (2014).

[13] D.K. Sut, An Application of Fuzzy Soft Relation in Decision Making Problems, International Journal of Mathematics Trends and Technology, Vol.3, No.2, (2012).

[14] K.V. Babitha, J.J. Sunil, Soft Set Relations and Functions, Comput. Math. Appl., Vol.60, pp.1840-1849, (2010).

[15] J.H. Park, O.H. Kim, Y.C. Kwun, Some properties of equivalence soft set relations, Comput. Math. Appl., Vol.63, pp.1079-1088, (2012).

[16] H. Yang, Z. Guo, Kernels and Closures of Soft Set Relations, and Soft Set Relation Mappings, Comput. Math. Appl., Vol.61, pp.651-662, (2011).

[17] B. Tanay, G. Yaylalı, New structures On Partially Ordered Soft Sets and Soft Scott Topology, Ann. Fuzzy Math. Inform., Vol.7, pp.89-97, (2014).

[18] M. Shabir, M. Naz, On soft topological spaces, Comput. Math. Appl., Vol.61, pp.1786-1799, (2011).

[19] N. Çağman, S. Karataş, S. Enginoğlu, Soft topology, Comput. Math. Appl., Vol.62, pp.351358, (2011).

[20] A. Aygünoğlu, H. Aygün, Some notes on soft topological spaces, Neural Comput. Appl., Vol.21, No.1, pp.113-119, (2012).

[21] I. Zorlutuna, M. Akdağ, W.K. Min, S. Atmaca, Remarks On soft topological spaces, Ann. Fuzzy Math. Inf., Vol.3, No.2, pp.171-185, (2012).

[22] S. Hussain, B. Ahmad, Some properties of soft topological spaces, Comput. Math. Appl., Vol.62, pp.4058-4067, (2011).

[23] B. Pazar Varol, H. Aygün, On soft hausdorf spaces, Ann. Fuzzy Math. Inf., Vol.5, No.1, pp.15-24, (2013).

[24] W.K. Min, A note on soft topological spaces, Comput. Math. Appl., Vol.62, pp.3524-3528, (2011).

[25] M. Shabir, M. Naz, On Bipolar Soft Sets, arXiv: 1303.1344v1 [math.LO], (2013).

[26] F. Karaaslan, S. Karatas, A new approach to bipolar soft sets and its applications, Discrete Math. Algorithm. Appl., 07, 1550054, (2015).

[27] M. Shabir, A. Bakhtawar, Bipolar soft connected, bipolar soft disconnected and bipolar soft compact spaces, Songklanakari J. Sci. Technol., Vol.39, No.3, pp.359-371, (2017).

[28] Y.T. Öztürk, On Bipolar Soft Topological Space, Journal of New Theory, Vol.20, pp.64-75, (2018).

(Naime Demirtaş) Mersin University, Faculty of Science and Art, Department of Mathematics, Mersin, Turkey

Email address, Naime DEMİTAŞ: naimedemirtas@mersin.edu.tr

(Orhan DAlKiliÇ) Mersin University, Faculty of Science and Art, Department of Mathematics, Mersin, Turkey

Email address, Orhan DALKILIÇ: orhandlk952495@hotmail.com 